\title{
Anreicherung des schweren Silberisotops durch Ionenwanderung in $\iota$-AgJ
}

\author{
Von Alfred KLEMM
}

\author{
Aus dem Kaiser-Wilhelm-Institut für Chemie, Tailfingen \\ (Z. Naturforschg. 2 a, 9-16 [1947]; eingegangen am 15. Aug. 1946)
}

\begin{abstract}
Festes $\alpha$-AgJ wurde bei $230^{\circ} \mathrm{C}$ zwischen einem Stück Kohle als Anode und einem Silberblech als Kathode während $20 \mathrm{Stdn}$. mit einer anodischen Stromdichte von $2 \mathrm{~A} / \mathrm{cm}^{2}$ elektrolysiert. Das nach der Elektrolyse übriggebliebene Silberjodid in der Nähe der Anode wurde massenspektrographisch untersucht. Unmittelbar an der Anode bestand das Silber aus $49 \%{ }^{107} \mathrm{Ag}$ und $51 \%{ }^{109} \mathrm{Ag}$. Die Zusammensetzung des Silbers ging mit zunehmendem Abstand von der Anode kontinuierlich in die natürliche $\left(52 \%{ }^{107} \mathrm{Ag}\right.$ und $48 \%{ }^{109} \mathrm{Ag}$ ) über, die ungefähr $2,3 \mathrm{~cm}$ von der Anode entfernt erreicht wurde. $Z$ wischen der relativen Massendifferenz der Silberionen und der relativen Differenz ihrer Wanderungsgeschwindigkeiten im $\alpha$-AgJ ergab sich daraus die Beziehung $\left(w_{1}-w_{2}\right) / \bar{w}$ $=-0,11\left(m_{1}-m_{2}\right) / \bar{m}$. Aus älteren, negativ verlaufenen Anreicherungsversuchen folgt für Ionenwanderung in Wasser: $\left(w_{1}-w_{2}\right) / \bar{w}<-0,018\left(m_{1}-m_{2}\right) / \bar{m}$.
\end{abstract}

$V_{t}$ ersuche, Isotope durch Ionenwanderung $\mathrm{zu}$ trennen, wurden 1921 von A. L in d e m a n $n^{1}$ angeregt und bald darauf in USA. ${ }^{2}$, Österreich ${ }^{3}$ und England ${ }^{4}$ durchgeführt. Diese Versuche blieben jedoch, wie J. Kendall 1942 der Royal Society zusammenfassend mitteilte, trotz großer experimenteller Anstrengungen ohne Erfolg. Die Gründe dafür waren folgende: 1 . Man verwandte wäßrige Lösungen, weil diese am bequemsten zu handhaben sind. Die Hydratisation der Ionen bewirkt aber eine Angleichung der Wanderungsgeschwindigkeiten. 2. Die Nachweismethode (chemische Atomgewichtsbestimmung) war nicht sehr genau.

Im Folgenden wird berichtet, wie durch Verwendung nichtsolvatisierter Ionen $\mathrm{Ag}^{+}$in $\alpha$ AgJ) und durch massenspektrographische Analyse erstmals eine Isotopentrennung durch Ionenwanderung nachweisbar wurde ${ }^{6}$.

Es sei daran erinnert, daß die Anreicherung von ${ }^{2} \mathrm{H}$, ${ }^{7} \mathrm{Li}$ und ${ }^{18} \mathrm{O}$ in Elektrolyserückständen nicht durch die Ionenwanderung im Elektrolyten, sondern durch den elektrodischen Abscheidungsmechanismus entsteht.

1 A. Lindemann, Proc. Roy. Soc. [London] Ser. A, 99, 102 [1921].

2 J. Kendall u. E. Crittenden, Proc. Nat. Acad. Sci. USA. 9, 75 [1923]; J. Ke nd a ll u. F. Wh it e, Proc. Nat. Acad. Sci. USA: 10, 458 [1924].

${ }^{3}$ M u rm a n n, Österr. Chemiker-Ztg. 26, 14 [1924].

4 G. Pill e y, Philos. Mag. J. Sci. 49, 892 [1925]; E. J e t te, Philos. Mag. J. Sci. 3, 258 [1927].
Dies folgt z. B. aus der Tatsache, daß die Deuteriumanreicherung nicht sehr davon abhängt, ob der Elektrolyt sauer oder alkalisch ist.

\section{Prinzip des Verfahrens}

Die $\alpha$-Phase des Silberjodids, deren Existenzbereich zwischen einem Umwandlungspunkt bei $145,8^{\circ} \mathrm{C}$ und dem Schmelzpunkt bei $502^{\circ} \mathrm{C}$ liegt, zeichnet sich dadurch aus, daß in ihr die Beweglichkeit der Ag+-Ionen von der gleichen Größenordnung wie in geschmolzenem $\mathrm{AgJ}$ ist (z. B. bei $\left.230^{\circ} \mathrm{C}: D_{\mathrm{Ag}}=2 \mathrm{~cm}^{2} / \mathrm{d}^{7}, x=1,7 \mathrm{Ohm}^{-1} \mathrm{~cm}^{-1}{ }^{8}\right)$. Spannt man eine Stange $\alpha$-AgJ zwischen eine Anode aus Kohle und eine Kathode aus Silber, so entwickeln sich an Anode und Kathode dem Strom äquivalente Mengen von Joddampf und Silber. Dabei wird das AgJ an der Anode scheinbar aufgezehrt, weil dort das Gitter der Jodionen, das dem $\alpha$-AgJ die Form gibt, abgebaut wird. Wenn nun ${ }^{107} \mathrm{Ag}+$ eine größere Beweglichkeit besitzt als ${ }^{109} \mathrm{Ag}+$, dann wird sich ${ }^{107} \mathrm{Ag}$ im kathodisch abgeschiedenen Silber und ${ }^{109} \mathrm{Ag}$ im anodennahen AgJ anreichern.

5 J. K en d a l l, Nature [London] 150, 136 [1942].

- A. K l e m m, Naturwiss. 32, 69 [1944] (kurze Originalmitteilg).

7 C. Tubandt, H. Reinhold u. W. Jost, Z. anorg. allg. Chem. 177, 253 [1929].

8 C. Tubandt u. E. Lor enz, Z. Physik. Chem. 87, 513 [1914]. 
Abschätzung der Trennwirkung

Wir geben Anreicherungen durch den Trennfaktor

$$
Q_{12}=n_{1} n_{2}^{0} / n_{2} n_{1}^{0}
$$

an. Es bedeutet: $n\left(\mathrm{~cm}^{-3}\right)$ Konzentration, 1 und 2 ${ }^{107} \mathrm{Ag}$ und ${ }^{109} \mathrm{Ag}, 0$ natürliches Gemisch. Die Wanderungsgeschwindigkeiten von ${ }^{107} \mathrm{Ag}^{+}$und ${ }^{109} \mathrm{Ag}+$ bezeichnen wir mit $w_{1}$ und $w_{2}$, die mittlere mit

$$
\bar{w}=\left(n_{1} w_{1}+n_{2} w_{2}\right) /\left(n_{1}+n_{2}\right) .
$$

Da das AgJ an der Kathode dauernd das natürliche Mischungsverhältnis $n_{1}^{0} / n_{2}^{0}$ behält, ist der durch den Unterschied der Wanderungsgeschwindigkeiten hervorgerufene Trennfaktor des abgeschiedenen Silbers $Q_{12}=w_{1} / w_{2}$. Er ist von der Elektrolysedauer unabhängig und unmeßbar klein.

Im Inneren des AgJ, dessen Querschnitt konstant sei, beginnt die Anreicherung des ${ }^{109} \mathrm{Ag}$ an der Anode und breitet sich von dort durch Diffusion in das AgJ hinein aus. Dieser als ,Stauung" bezeichnete Vorgang wurde in einer früheren Arbeit ${ }^{9}$ berechnet. Wir wollen ihn hier etwas vereinfacht betrachten: Aus der genauen Rechnung entnehmen wir, daß sich der Konzentrationsabfall nach einer Elektrolysedauer $t$ von $x=0$ bis ungefähr $x=\sqrt{\pi D t}$ erstreckt ( $x=\mathrm{Ab}$ stand von der Anode). Dies ist plausibel, da Diffusionslängen immer von der Größenordnung $\sqrt{ }$ It sind. Nun gilt offenbar

$$
\int_{0}^{\sqrt{\pi D t}} n_{1,2} d x=\bar{n}_{1,2} \sqrt{\pi D t}=n_{1,2}^{0}\left(\sqrt{\pi} D t+v t-w_{1,2} t\right) \text {, }
$$

denn die Menge ${ }^{107,109} \mathrm{Ag}$ links einer Stelle im Jodgitter ist gleich der Menge, die zur Zeit $t=0$ links war, minus der Menge, die in der Zeit $t$ vorbeigewandert ist. Aus (1) ergibt sich

$$
Q_{21}=\frac{\bar{n}_{2} n_{1}^{0}}{\overline{n_{1}} n_{2}^{0}} \approx 1+\frac{\bar{w} t}{\sqrt{\pi D t}} \frac{w_{1}-w_{2}}{\bar{w}} .
$$

Die „Entmischbarkeit“ $\left(w_{1}-w_{2}\right) / w$ ist eine Konstante, während die inittlere "Vervielfachung“ $\bar{w} t / \sqrt{\pi D t}$ um so größer ist, je größer die Strom-

${ }_{9}$ A. K l e m m, Z. Naturforschg. 1, 252 [1946].

${ }^{10} \mathrm{C}$. Tubandt u. S. Eg gert, Z. anorg. allg. Chem. 110, 196 [1920]. dichte $i$ und die Elektrolysedauer $t$ sind. Es hat sich gezeigt, daß zur Erreichung einer bequem nachweisbaren Isotopenanreicherung. eine Stromdichte von $2 \mathrm{~A} / \mathrm{cm}^{2}$ bei $20 \mathrm{Stdn}$. Elektrolysedauer ausreicht. Dabei wird eine Silberjodidsäule der Länge $\bar{w} t=65 \mathrm{~cm}$ zersetzt, und die Anreicherung ist bei $230^{\circ} \mathrm{C}\left(D_{\mathrm{Ag}}=2 \mathrm{~cm}^{2} / \mathrm{d}\right)$ innerhalb eines Anodenabstandes von $\sqrt{\pi D t}=2,3 \mathrm{~cm} \mathrm{zu}$ suchen.

Darstellung des Silberjodids

Für den Hauptversuch wurden ungefähr $270 \mathrm{~g} \mathrm{AgJ}$ benötigt.

Das Ziel der Darstellung von Silberjodid für diese Versuche ist die Gewinnung eines Präparates, das eine möglichst geringe Neigung zur Bildung von Silberdendriten aufweist. Das Silberjodid muß deshalb frei von allen Beimengungen sein, die beim Erhitzen eine Zersetzung in Silber und Jod begünstigen. Man hat demnach hauptsächlich darauf zu achten, daß das Präpazat nur so viel Silber enthält, als dem stöchiometrischen Verhältnis entspricht, und daß in das Präparat keine organischen Bestandteile, z. B. Staubfäden oder Gummiteilchen, gelangen. Darstellungsvorschriften findet man bei C. Tuband und E. L or en $z^{8}$, C. T u b a n d t und S. E g g e r t ${ }^{10}$ sowie bei E. C o h e n und D. van Dobbenburgh ${ }^{11}$.

Man fällt das Silberjodid bei dunkelrotem Licht durch Zusammengeben von wäßrigen Silbernitratund Kaliumjodidlösungen. Da das Silberjodid aus der Lösung Silberionen adsorbiert, muß angestrebt werden, daß es möglichst wenig mit Silberionen in Berührung kommt. Dies wird dadurch erreicht, daß man 1. in verdünnter Lösung (ca. $0,01 \mathrm{~g} / \mathrm{cm}^{3}$ ) fällt, 2. die Silbernitratlösung langsam unter heftigem Rühren in die Kaliumjodidlösung einfließen läßt und 3. einen 0,5 -proz. Überschuß an Kaliumjodid übrigläßt. Infolge des Kaliumjodidüberschusses bildet sich allerdings eine milchige Emulsion ${ }^{12}$, die man aber durch Zugabe von Salpetersäure peptisieren kann. Der Niederschlag wird vom Kaliumnitrat getrennt, indem man 12-mal dekantiert, die Flüssigkeitsmenge mit destilliertem Wasser ergänzt und kräftig durchschüttelt. Nötigenfalls wird die Koagulation durch Zugabe weiterer Salpetersäure beschleunigt. Die Fällung von $350 \mathrm{~g}$ Silberjodid geschah mit $20 l$ Wasser, und dann wurden zum Auswaschen des Niederschlags noch einmal $25 l$ Wasser verbraucht. Der Niederschlag wird im elektrischen Trockenschrank bei bis $200^{\circ} \mathrm{C}$ gesteigerter Temperatur getrocknet. Es entsteht ein mehr oder weniger harter Kuchen, den man im Achatmörser zu einem feinen Pulver zerstößt. Es ist darauf zu achten, daß keine Glas- oder Porzellansplitter in das Präparat gelangen, weil diese sich bei der Elektrolyse an der Anode ansammeln und dort stören.

11 E. Cohen u. D. van Dobbenburgh, Z. physik. Chem. 137, 289 [1928].

${ }_{12}$ A. Lottermoser, Z. physik. Chem. 70, 239 [1910]. 
Anode und Trennsäule

In einer Handpresse wurden aus AgJ-Pulver etwa 250 Pastillen von $3,9 \mathrm{~mm} \varnothing$ und ca. $3 \mathrm{~mm}$ Höhe hergestellt. Diese Pastillen wurden in einem Rohr aus Jenaer Geräteglas von genau $4 \mathrm{~mm}$ Innen- $\varnothing$ (KPG.Rohr) und ca. $9 \mathrm{~mm}$ Außen- $\varnothing$ untergebracht. Beim Erwärmen auf $230^{\circ} \mathrm{C}$ und Zusammenpressen mit $5 \mathrm{~kg} / 0,126 \mathrm{~cm}^{2}$ schmiegten sich die Pastillen fest aneinander und gegen die Glaswand, wobei sich die ursprünglich $74 \mathrm{~cm}$ lange Säule um $1 \mathrm{~cm}$ verkürzte und ein weiteres Verschieben der Pastillen im Rohr unmöglich wurde. Beim Erkalten nach der Elektrolyse sprengte das Silberjodid wegen seiner Volumenvergrößerung beim Unterschreiten von $145,8^{\circ} \mathrm{C}$ das Glasrohr.

Die Kohle konnte wegen der Gefahr des Hängenbleibens nicht ganz stramm in das Glasrohr eingepaßt werden, und es blieb deshalb bei der Elektrolyse eine dünne Schicht von Silberjodid zwischen Glas und Kohle stehen. Beim endgültigen Versuch wog diese Schicht ca. $75 \mathrm{mg}$, also nur 4,5\% der 1,65 g AgJ des 2,3 cm langen angereicherten Stückes. Die entwickelten Joddämpfe schlugen sich in den kälteren Teilen des Ofenrohres in Form von Kristallen nieder. An der Stirnfläche der Kohle (Homogenkohle A der Firma Siemens Plania) fand man nach 20 Stdn. Betrieb eine dünne Schicht von schwarzem Staub.

Gelegentlich traten Funken und Stromunterbrechung an der Anode auf (sogenannter Anodeneffekt) und beendeten den Versuch vorzeitig, weil das Glas durch die Hitze des Lichtbogens angegriffen wurde und die Kohle sich festfraß. Die Ursachen des Anodeneffektes waren vermutlich Verunreinigungen im Silberjodid oder Hemmungen beim Nachschieben der Kohle.

\section{$\mathrm{K}$ a thode}

Als Kathodenvorgang wurde die Abscheidung von Silber aus $\alpha$-AgJ auf Silberblech gewählt. Diese Abscheidung geschieht in Form von Silberfäden, die bei niedriger Stromdichte einen dichten $\mathrm{Pelz}$ von einheitlicher Fadenlänge bilden, der sich zwischen das Silberblech und das Salz drängt. Bei hoher Stromdichte wachsen einzelne Fäden in Richtung der Stromlinien durch das Salz hindurch und beenden die Elektrolyse, wenn sie bis zur Anode vorgedrungen sind. Die Ursache dieser „Dendriten“ ist die erhöhte Stromdichte an den Spitzen der längsten Fäden.

Zur Verminderung der kathodischen Stromdichte wurde der Strom dem Silberblech über einen Schutzzylinder aus AgJ von $3 \mathrm{~cm} \varnothing$ und 2,5 cm Höhe zugeführt ${ }^{13}$. Die 56-fache Querschnittserweiterung verminderte die Stromdichte von $2 \mathrm{~A} / \mathrm{cm}^{2}$ an der Anode auf $35,5 \mathrm{~mA} / \mathrm{cm}^{2}$ an der Kathode. Der Schutzzylinder wurde aus $105 \mathrm{~g} \mathrm{AgJ-Pulver} \mathrm{durch} \mathrm{Pressen} \mathrm{in} \mathrm{einer}$ stählernen Hohlform mit $1 \mathrm{t} / \mathrm{cm}^{2}$ Druck hergestellt.

13 Für die Mitteilung, daß die Tendenz zur Dendritbildung bei kleiner Stromdichte gering ist, danke ich Hrn. Prof. C. W a g n e r.

\section{Vorversuch}

Abb. 1 zeigt schematisch die Anordnung des Versuches. Die Stromstärke wurde durch den Schiebewiderstand $W$ auf $250 \mathrm{~mA}$ gehalten, und der Abstand $a$ zwischen Kohle und Schutzzylin-

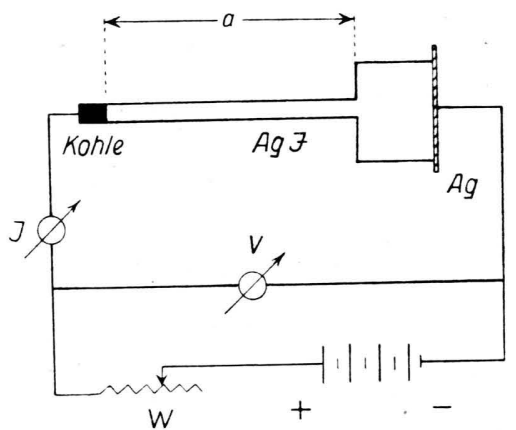

Abb. 1. Anordnung des Vorversuchs.

der sowie die Elektrolysespannung $V$ wurden als Funktion der Zeit $t$ gemessen und in Abb. 2 aufgetragen.

Nach anfänglichen Schwankungen verminderte sich $V$ gleichmäßig infolge der Verkürzung von

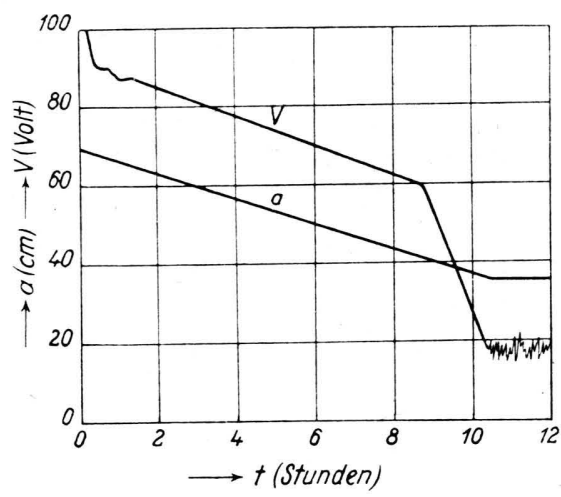

Abb. 2. Gemessene Elektrolysespannung $V$ und Länge der Trennsäule $a$ als Funktionen der Zeit $t$ beim Vorversuch.

a. Zur Zeit $t=8.75 \mathrm{~h}$ wurde der Abfall von $V$ plötzlich steiler, weil ein Dendrit den Schutzzylinder durchwachsen hatte und nun während 1,75 Stdn. mit konstanter Geschwindigkeit in der engen Silberjodidsäule vordrang. 10,5 Stdn. nach Elektrolysebeginn hatte der Dendrit bei einer Säulenlänge $a=36 \mathrm{~cm}$ die Anode erreicht. Die Zersetzung von AgJ hörte jetzt auf, weil die Elektronen nun vom Dendriten in die Kohle über- 
traten; allerdings war der Kontakt schlecht, daher die Spannungsschwankungen.

Aus Abb. 2 kann man folgende Zahlenwerte entnehmen: Die Wanderungsgeschwindigkeit der Silberionen war $\bar{w}=-d a / d t=3,17 \mathrm{~cm} / \mathrm{h}=8,8$ $\cdot 10^{-4} \mathrm{~cm} / \mathrm{sec}$. Daraus folgt mit $i=1,98 \mathrm{~A} / \mathrm{cm}^{2}$ und $F=96500$ Coulomb $: c=i / F \bar{w}=0,023 \mathrm{~mol} / \mathrm{cm}^{3}$. Der Literaturwert ${ }^{11}$ für die Dichte bei $230^{\circ} \mathrm{C}$ ist ungefähr $\varrho=5,87 \mathrm{~g} / \mathrm{cm}^{8}$, also mit $\mathrm{M}=234,8$ : $c=0,025 \mathrm{Mol} / \mathrm{cm}^{3}$. Ohne Dendrit ist nach Abb. 2 $d V / d t=-3,75 \mathrm{Volt} / \mathrm{h}$. Die Feldstärke war also $E=(d V / d t) /(d a / d t)=1,18 \mathrm{Volt} / \mathrm{cm}$ und die Leitfähigkeit $x=i / E=1,68(\mathrm{Ohm} \mathrm{cm})^{-1}$. Der Literaturwert ${ }^{8}$ bei $230^{\circ} \mathrm{C}$ ist $x=1,7(\mathrm{Ohm} \mathrm{cm})^{-1}$.

Nach Abb. 2 ist die Geschwindigkeit des Dendritenwachstums in der engen AgJ-Säule gleich $20,6 \mathrm{~cm} / \mathrm{h}$. Das Gewicht des Dendriten pro Längeneinheit berechnet man daraus $\mathrm{zu} 49 \mathrm{mg} / \mathrm{cm}$ und die Feldstärke in der den Dendriten enthaltenden Silberjodidsäule zu 0,12 Volt/cm. Der Silberpelz zwischen Silberblech und Schutzzylinder hatte nach 10 Stdn. Elektrolyse eine Höhe von etwa $9 \mathrm{~mm}$. Einige wenige Fäden waren weit in den Schutzzylinder eingedrungen, davon einer in die Trennsäule.

\section{H a p tversuch}

Der Vorversuch hatte gelehrt, daß ein einziger Schutzzylinder zur Aufnahme des in 20 Stdn. abzuscheidenden Silbers nicht ausreichte. Deshalb wurde die Apparatur in zwei Teile zerlegt, einen Anodenteil und einen auswechselbaren Kathodenteil, wie es Abb. 3 zeigt. Das Eindringen eines Dendriten in die Signalsäule war am schnelleren

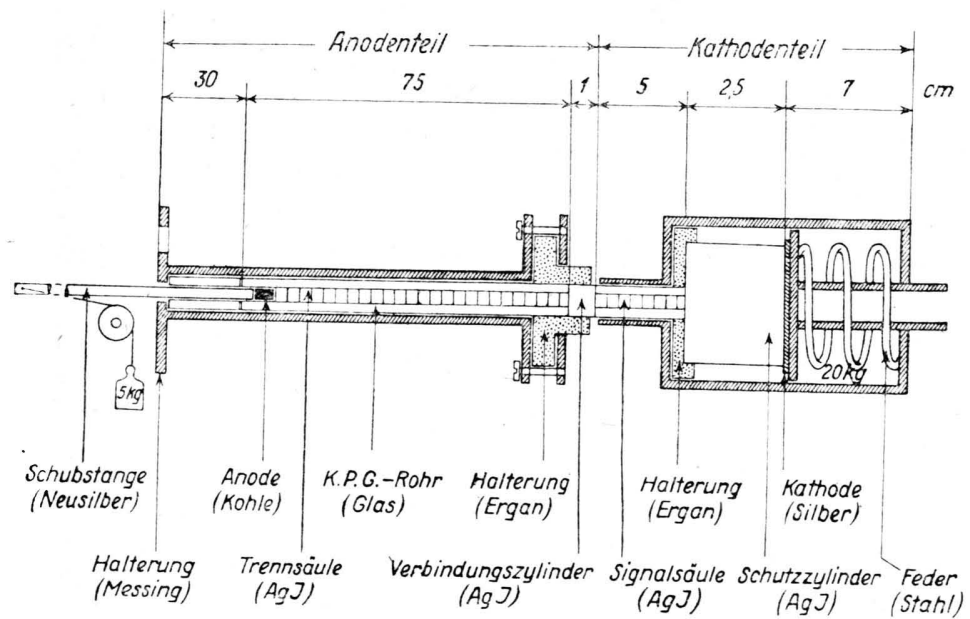

Abb. 3. Endgültige Versuchsanordnung. zeitlichen Abfall der Elektrolysespannung zu erkennen, und man konnte dann rechtzeitig den verbrauchten Kathodenteil durch einen neuen ersetzen, der in einem zweiten Ofen bereitgehalten wurde. Beim Hauptversuch wurde der Kathodenteil 10,7 Stdn. nach Elektrolysebeginn ausge wechselt.

Die Gesamtdauer des Versuchs betrug 20 Stdn., die Stromstärke $250 \mathrm{~mA}$. Dabei wurde eine Silberjodidsäule von $65 \mathrm{~cm}$ Länge abgebaut. Am Schluß des Versuches war der Abstand zwischen Kohle und Verbindungszylinder $8 \mathrm{~cm}$.

Erläuterungen zu Abb. 3: Ergan ist ein keramisches Material, das man mit harten Stählen bearbeiten kann. Die Stahlfeder übte eine Kraft von ca. $20 \mathrm{~kg}$ aus. Die Schubstange hatte $3,6 \mathrm{~mm} \varnothing$. Verschiebbare EisenKonstantan-Thermoelemente wurden im Anodenteil oberhalb der Trennsäule und im Kathodenteil im Federraum eingeführt.

Der Ofen bestand aus einem horizontal gelegenen Porzellanrohr von $1,5 \mathrm{~m}$ Länge und $5 \mathrm{~cm}$ lichter Weite, das auf 1,1 m Länge mit 55 Windungen Chromnickelband $\left(2 \cdot 0,3 \mathrm{~mm}^{2}\right)$ bewickelt und durch eine Kieselgurschicht von $5 \mathrm{~cm}$ Dicke nach außen thermisch isoliert war. Durch vier Abgriffe wurde die Wicklung in drei Abschnitte geteilt, so daß durch schwächere Beheizung des mittleren, $74 \mathrm{~cm}$ langen Wicklungsabschnittes eine gleichmäßige Temperaturverteilung eingestellt werden konnte. Zur Erzeugung von $230^{\circ} \mathrm{C}$ nahm der Ofen ca. 100 Watt auf.

Messung der Isotopenanreicherung

Ein an die Anode angrenzendes 3,6 cm langes Stück der Silberjodidsäule wurde nach Entfernung des Glases durch Schnitte senkrecht zur Achse in 12 Portionen geteilt. Die AgJ-Portionen wurden in verd. Schwefelsäure mit Zinkstaub in Berührung gebracht, und das entstandene Silber wurde zur Entfernung von überschüssigem Zink mit Schwefelsäure versetzt, dann mit Wasser gewaschen, getrocknet und zu Tabletten gepreßt.

Von fünf dieser Tabletten sowie natürlichem Silber wurden mit dem aus Wien stammenden M a t t a u chHer z o g schen Massenspektrographen des Instituts etwa 200 Massenspektrogramme auf Schumann-Platten (Agfa) hergestellt. Die Ionen wurden nach Dempster durch eine Funkenstrecke im Hochvakuum erzeug,, wobei die Silbertablette eine der Elek- 
troden war $^{14}$. Die Expositionszeiten lagen zwischen 2 und 40 sec.

Da bei den verwendeten S chu mann-Platten die Schwärzungskurve (Schwärzung $s$ als Funktion der eingestrahlten Ionenmenge $n$ ) zuweilen von Platte $\mathrm{zu}$ Platte eine andere Gestalt besitzt und alle Einzelmessungen wegen der Untergrundschwankungen ziemlich stark streuen, macht die Auswertung der Aufnahmen gewisse Schwierigkeiten.

Mit dem $Z$ ei $ß$ schen Registrierphotometer wurden von jeder Silberprobe etwa 30 Schwärzungsverhältnisse $s_{2} / s_{1}$ gemessen und als Funktion von $s_{1}$ aufgetragen $\left(s_{1,2}\right.$ ist der Ausschlag vom Grundschleier aus, den das Photometer beim Passieren der Linienmitte zeigt, dividiert durch den Ausschlag bei Schließung des Photometerspaltes). Durch die so entstehende, stark streuende Punkteschar wurde eine Gerade gelegt (infolge der Streuung war es nicht möglich, eine willkürfreie gekrümmte Kurve durch die Punkte zu legen), deren Ordinatenabschnitt als das gesuchte Mischungsverhältnis $n_{2} / n_{1}$ angesehen wurde.

Die gemessenen Schwärzungsverhältnisse sind für natürliches Silber als Punkte und für die am meisten angereicherte Probe als Kreise in Abb. 4 eingezeichnet. Die schrägen Geraden in Abb. 4 zeigen die geradlinige Extrapolation auf $s_{1}=0$, durch die sich das Mischungsverhältnis bei natürlichem Silber zu 0,92 (52\% ${ }^{107} \mathrm{Ag}$ und $\left.48 \%{ }^{109} \mathrm{Ag}\right)$ und bei der besten Probe zu 1,04 (49\% ${ }^{107} \mathrm{Ag}$ und $51 \%{ }^{109} \mathrm{Ag}$ ) ergab. Analog wurden die Mischungsverhältnisse der vier anderen Proben gemessen.

Natürliches Silber besitzt auch nach massenspektrometrischen Messungen von W. $\mathrm{Paul}{ }^{15}$ das Mischungsverhältnis $\mathrm{n}_{2}^{0} / n_{1}^{0}=0,92$. A. F l a m mersfeld und O.Brun a ${ }^{16}$ haben natürliches Silber und meine beiden am meisten angereicherten Silberproben mit langsamen Neutronen aktiviert und haben in quantitativer Ubereinstimmung

14 S. J. M a t tauch u. H. Ew ald, Z. Physik 122, 314 [1944].

15 W. P a u l, Naturwiss. 31, 419 [1943].

16 A. F l a m m e r s f e l d u. O. B r un a, Naturwiss. 32. 70 [1944]. mit den massenspektrographischen Ergebnissen festgestellt, daß die vom ${ }^{110} \mathrm{Ag}$ stammende 24 -secAktivität im Vergleich zur 2,4-min-Aktivität des ${ }^{108} \mathrm{Ag}$ bei den angereicherten Proben entsprechend stärker auftritt als bei natürlichem Silber. 
Berechnung des Masseneffektes in $x-A g J$ und in $\mathrm{H}_{2} \mathrm{O}$ aus den Versuchsdaten

Nach der Phänomenologie des Stauverfahrens ${ }^{9}$ gilt für kleine Anreicherungen

$$
\begin{gathered}
Q_{21}=\frac{n_{2} n_{1}^{0}}{n_{1} n_{2}^{0}}=1+f a_{12}, \\
f=\frac{2 \bar{w} t}{\sqrt{\pi D t}} \eta(\iota), \\
a_{1,2}=\frac{w_{1}-w_{2}}{\bar{w}}=\mu \frac{m_{1}-m_{2}}{\bar{m}}, \\
\psi(\diamond)=e^{-\iota^{2}}-\sqrt{\pi \zeta}\left(1-\frac{2}{\sqrt{\pi}} \int_{0}^{\zeta}-a^{2} d c\right), \\
\zeta=x / 2 \sqrt{D t} .
\end{gathered}
$$

Mit $D=2 \mathrm{~cm}^{2} / \mathrm{d}, t=0,83 \mathrm{~d}$ und $\bar{w} t=65 \mathrm{~cm}$ ergibt sich als Vervielfachung $f=56 \psi(0,39 x)$. Die beste Übereinstimmung zwischen dem theoretischen und dem experimentellen Verlauf von $Q_{21}$ erreicht man dann, wenn man als Entmischbarkeit $\alpha_{12}=0,002$ setzt. Die so berechnete Funktion $Q_{21}-1$ ist in Abb. 5 eingezeichnet. Mit $m_{1}=107$, $m_{2}=109$ und $\bar{m}=108$ folgt für den Masseneffekt $\mu=-0,11$.

Zum Vergleich wollen wir versuchen, etwas über den Masseneffekt der Ionenwanderung in Wasser zu erfahren, und ziehen hierfür folgende Angaben aus den unter ${ }^{2}$ bis ${ }^{5}$ zitierten Arbeiten heran:

Ein horizontales Glasrohr wurde mit AgarAgar-Gel gefüllt, das links Na-Acetat, in der Mitte $\mathrm{NaCl}(0,1-n$.$) und rechts \mathrm{NaOH}$ enthielt. Durch ein elektrisches Feld wurden die Acetat-, Chlorund Hydroxylionen in 100 Tagen mit der mittleren Wanderungsgeschwindigkeit $\bar{w}=30 \mathrm{~cm} / \mathrm{d}$ um $30 \mathrm{~m}$ nach rechts bewegt. Die NaCl-Zone behielt dabei ihre Länge bei und blieb scharf begrenzt. Es wurde dann von der NaCl-Zone vorn und hinten je ein $1 \mathrm{~cm}$ langes Stück abgeschnitten und das Atomgewicht des Chlors in beiden Stücken auf 0,1\% genau bestimmt. Eine Anreicherung von ${ }^{35} \mathrm{Cl}$ im vorderen und von ${ }^{37} \mathrm{Cl}$ im hinteren Stück wurde nicht gefunden. Ähnliche Versuche mit Mg verliefen ebenfalls negativ. So viel aus den unter ${ }^{2}$ bis ${ }^{5}$ zitierten Arbeiten.

Einsetzen von $\left(M-M^{0}\right) / M^{0}=-0,001, M^{0}=35,46$, $m_{1}=35$ und $m_{2}=37$ in die für schwach angereicherte Isotopengemische gültige Gleichung

$$
Q_{12}=1+\frac{M-M^{0}}{M^{0}} \frac{M^{0}\left(m_{2}-m_{1}\right)}{\left(M^{0}-m_{1}\right)\left(M^{0}-m_{2}\right)}
$$

ergibt $Q_{1_{2}}=1,1$. Aus $t=100 \mathrm{~d}, \bar{w} t=3000 \mathrm{~cm}$ und $D=1 \mathrm{~cm}^{2} / \mathrm{d}$ folgt ferner $\sqrt{\pi D t}=18 \mathrm{~cm}$ und $f_{0}=2 \bar{w} t / \sqrt{\pi D t}=340$. Setzen wir vorsichtshalber $\ddot{r}_{0}>100$ und $Q_{0}<1,1$ in $Q_{0}=1+f_{0} \alpha$ ein, so ergibt sich $\alpha<0,001$. Daraus folgt $|\mu|<0,018$.

Der Masseneffekt der Ionenwanderung ist also in $\alpha$-AgJ absolut gleich 0,1 und in wäßrigen Lösungen mindestens 5-mal klëner.

\section{Zur Kinetik der Ionenwanderung}

Nach L. Strock ${ }^{17}$ ist im $\alpha$-AgJ die Zahl der vom $\mathrm{Ag}^{+}$zeitweise besetzten Gitterplätze 21-mal größer als die Zahl der vorhandenen $\mathrm{Ag}^{+-I o n e n .}$ Die einem Ag+-Ion benachbarten Gitterplätze sind also meistens frei.

Wenn man nun das $\alpha$-AgJ als starres periodisches Gitterpotential mit darin unabhängig voneinander schwingenden und springenden $\mathrm{Ag}^{+}$Ionen auffassen dürfte, dann wäre $\mu=-0,5 \mathrm{zu}$ erwarten, denn die der Wanderungsgeschwindigkeit proportionale Platzwechselwahrscheinlichkeit wäre der Schwingungsfrequenz und damit der reziproken Wurzel aus der Ag+-Masse proportional. Der Befund $\mu=-0,1$ zeigt, daß diese Betrachtungsweise $\mathrm{zu}$ primitiv ist.

Man wird berücksichtigen müssen, daß die Frequenz $v_{1,2}$ durch das Mitschwingen der Umgebung kleiner ist als bei obigem Idealfall. Statt $m_{1,2}$ wird also eine effektive Masse $m_{1,2}^{*}>m_{1,2}$ auftreten, wobei $m_{2}^{*}-m_{1}^{*}=m_{2}-m_{1}$. Außerdem zieht vielleicht jeder spontane Sprung, dessen Wahrscheinlichkeit massenabhängig ist, $z$ massenunabhängige Sprünge benachbarter Ionen in gleicher Richtung nach sich. Der Einfluß dieser beiden Effekte auf den Masseneffekt wäre durch die Gleichung

$$
\mu=-0,5 \frac{m}{m^{*}} \frac{1}{1+z}
$$

wiederzugeben, wobei unser Wanderungsversuch in $\alpha$-AgJ aussagt

$$
\frac{m}{m^{*}} \frac{1}{1+z}=\frac{1}{5} .
$$

17 L. S t r o ck, Z. Kristallogr. 93, 285 [1936]. 
Wenn man außer dem Verhältnis der Wanderungsgeschwindigkeiten $w_{1} / w_{2}=1,002$ im natürlichen $\alpha$-AgJ die Leitfähigkeiten $x_{1}$ und $x_{2}$ der Reinsalze $\alpha^{-107} \mathrm{AgJ}$ und $\alpha^{-109} \mathrm{AgJ}$ kennen würde, dann könnte man $z$ durch Einsetzen von $x_{1} / x_{2}$ $=v_{1} / \nu_{2}$ in die Gleichung

$$
\frac{w_{1}}{w_{2}}=\frac{v_{1}+\gamma_{1} z v_{1}+\gamma_{2} z v_{2}}{v_{2}+\gamma_{1} z v_{1}+\gamma_{2} z v_{2}}
$$

bestimmen, wo $\gamma_{1}$ und $\gamma_{2}$ die Molenbrüche im natürlichen $\alpha$-AgJ sind.

Ein ähnlicher Fall liegt bei Silber- und Kupferjodid vor: Das Verhältnis der Wanderungsgeschwindigkeiten von $\mathrm{Ag}^{+}$und $\mathrm{Cu}^{+}$in dem Mischkristall $\alpha-\mathrm{AgCuJ}_{2}$ ist 1,87 , während das Verhältnis für die Reinsalze $\alpha$-Ag.J und $\alpha$-CuJ den Wert 2,95 hat ${ }^{18}$. Einsetzen von $w_{1} / w_{2}=1,87, v_{1} / \nu_{2}=2,95$ und $\gamma_{1}=\gamma_{2}=0,5$ in (10) ergibt $z=0,63$. Man darf also vielleicht annehmen, $\operatorname{daß}$ im $\alpha-\mathrm{AgCuJ}_{2}$ jedes spontan springende Kation die benachbarten Kationen im Mittel so verschiebt, als würde einer dieser Nachbarn einen 0,63-mal kürzeren Sprung in der gleichen Richtung machen.

Selbstverständlich sind die hier gebrachten Überlegungen nicht endgültig, sondern müssen nach Vermehrung des Versuchsmaterials ausgebaut werden.

Theoretische Rechtfertigung derangewandten photometrischen Methode

Wir untersuchen $s_{2} / s_{1}$ als Funktion von $s_{2}$ bei konstantem Mischungsverhältnis $n_{2} / n_{1}$ für die zwei typischen Schwärzungskurven

$$
\begin{array}{ll}
s=1-\exp (-n) & \text { (Fall I), } \\
s=1-(1+n) \exp (-n) & \text { (Fall II). }
\end{array}
$$

( $s$ und $n$ sind Maße für Schwärzung und Ionenmenge.)

Abb. 6 zeigt diese beiden Schwärzungskurven. (I) gilt, wenn schon ein einziger Treffer zur Schwärzung eines Kornes genügt, während (II) gilt, wenn zwei Treffer für die Schwärzung eines Kornes erforderlich sind. Dies läßt sich leicht nachweisen:

Ein Stück der Platte enthalte eine Anzahl gleichartiger Körner und werde mit $n$ Trefferchancen, die wir kurz „Schüsse“ nennen wollen, belegt. Als Treffer bezeichnen wir ein Ereignis, das im Fall I allein, im Fall II zusammen mit einem zweiten solchen Ereignis zur Schwärzung des Kornes genügt. $p$ sei die Wahrscheinlichkeit, daß ein bestimmtes Korn von einem bestimmten Schuß getroffen wird; die Wahrscheinlichkeit, daß es nicht getroffen wird, ist dann $1-p$.

18 C. Tubandt, H. Reinhold u. W. Jost, Z. Physik. Chem. 129, 69 [1927].
Wir fragen nach der Wahrscheinlichkeit $w_{a}$, daß ein Korn $\alpha$ Treffer und $(n-\alpha)$ Nichttreffer erhält.

Denkt man sich die Schüsse numeriert, so ist die Wahrscheinlichkeit, daß $\alpha$ bestimmte Nummern Treffer und $(n-\alpha)$ bestimmte Nummern Nichttreffer sind, gleich $p^{a}(1-p)(n-a)$. Die Zahl der denkbaren Verteilungen von $\alpha$ Treffern auf $n$ Nummern ist

Also ist

$$
\frac{n !}{a !(n-a) !}=\left(\begin{array}{l}
n \\
a
\end{array}\right) \text {. }
$$

$$
w_{a}=\left(\begin{array}{l}
n \\
a
\end{array}\right) p^{a}(1-p)^{n-a} .
$$

Für $p \ll 1$ und $\alpha \ll n$ gelten die Näherungen $(1-p)^{(n-a)}=e^{-p n}$ sowie $\left(\begin{array}{l}n \\ a\end{array}\right)=\frac{n^{a}}{a !}$, und man erhält

$$
w_{a}=\frac{(p n)^{a}}{c !} \exp (-p n) .
$$

Die Wahrscheinlichkeit $w_{\text {I }}$ für mindestens einen Treffer $(\alpha \geqq 1)$ ist dann

$$
w_{\mathrm{I}}=1-w_{0}=1-\exp (-p n)
$$

und für mindestens zwei Treffer $(\alpha \geqq 2)$

$$
w_{\mathrm{II}}=1-w_{0}-w_{1}=1-(1+p n) \exp (-p n) .
$$

Wir deuten $w_{\mathrm{I}, \mathrm{I}}$ als die Zahl der mindestens 1,2-mal getroffenen Körner, dividiert durch die Gesamtzahl der Körner, und setzen demnach $w_{\mathrm{I}, \mathrm{II}}=s_{\mathrm{I}, \mathrm{II}} \cdot p n$ ist dem Ionenstrom proportional.

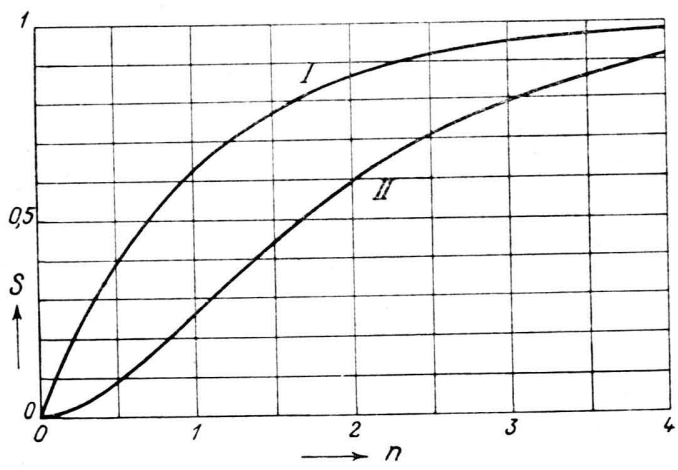

Abb. $\dot{6}$. Die Schwärzungskurven $s=1-\exp (-n)$ (I) und $s=1-(1+n) \exp (-n)$ (II).

H. Lichtblau ${ }^{19}$ hat bei empfindlichen Schu m a n n - Platten (Q3-Platten der Ilford) Schwärzungskurven vom Typ I nachgewiesen, während er für unempfindliche. S c h u m a n n - Platten (Q1 - Platten der Ilford) Schwärzungskurven vom Typ II fand. Bei empfindlichen Platten genügt also ein Treffer zur Schwärzung des Kornes, bei unempfindlichen nicht. Die zur Untersuchung der Silberproben benutzten

19 H. L i c h t b l a u, Physik. Z. 41, 82 [1940]. 
Platten (Schumann hart der Agfa) waren empfindlich, und Matta $\mathrm{ch} \mathrm{c}$. Ew ald haben entsprechend bei diesem empfindlichen Plattenmaterial nie einen Wendepunkt oder Schwellenwert in den Schwärzungskurven gefunden.

Zur Vereinfachung der Darstellung werden wir nicht $s_{2} / s_{1}$, sondern $\left(s_{2} / s_{1}-1\right) /\left(n_{2} / n_{1}-1\right)$ als Funktion von $s_{2}$ betrachten, was an der Gestalt der Kurve nichts ändert, da $n_{2} / n_{1}$ konstant ist.

Für Fall I hat man

a) für $n_{1} \approx n_{2}$ :

$$
\left(\frac{s_{2}}{s_{1}}-1\right) /\left(\frac{n_{2}}{n_{1}}-1\right) \approx \frac{n}{s} \frac{d s}{d n}=\frac{s-1}{s} \ln (1-s),
$$

b) für $n_{1} \ll n_{2}$ :

$$
\left(\frac{s_{2}}{s_{1}}-1\right) /\left(\frac{n_{2}}{n_{1}}-1\right) \approx \frac{s_{2}}{n_{2}}=-\frac{s_{2}}{\ln \left(1-s_{2}\right)} .
$$

Für Fall II ergibt sich

a) für $n_{1} \approx n_{2}$ :

$\left(\frac{s_{2}}{s_{1}}-1\right) /\left(\frac{n_{2}}{n_{1}}-1\right) \approx \frac{n^{2} \exp (-n)}{1-(1+n) \exp (-n)}$,

b) für $n_{1} \ll n_{2}$ :

$\left(\frac{s_{2}}{s_{1}}-1\right) /\left(\frac{n_{2}}{n_{1}}-1\right)=\infty$.

Abb. 7 zeigt für die Fälle Ia, Ib, II a und IIb die Funktion $\left(s_{2} / s_{1}-1\right) /\left(n_{2} / n_{1}-1\right)$ in Abhängigkeit von $s_{2}$. In Fall I wird für $s_{2}=0$ das Schwärzungsverhältnis mit dem Mischungsverhältnis identisch, in Fall II dagegen nicht. Verallgemeinernd kommen wir zu dem Ergebnis, daß das Verfahren der Extrapolation auf die Schwärzung Null nur anwendbar ist, wenn die Schwärzungskurve nicht ,durchhängt“, wenn sie also weder einen Wendepunkt noch einen Schwellenwert besitzt. Da bei den verwendeten S c h u man n - Platten diese Bedingung erfüllt war, konnte das besprochene Méßverfahren angewandt werden.

Weiter sehen wir aus Abb.7, daß in Fall I für alle Mischungsverhältnisse der Verlauf von $s_{2} / s_{1}$ zwischen $s_{2}=0$ und $s_{2}=0,5$ wenig gekrümmt ist, so daß

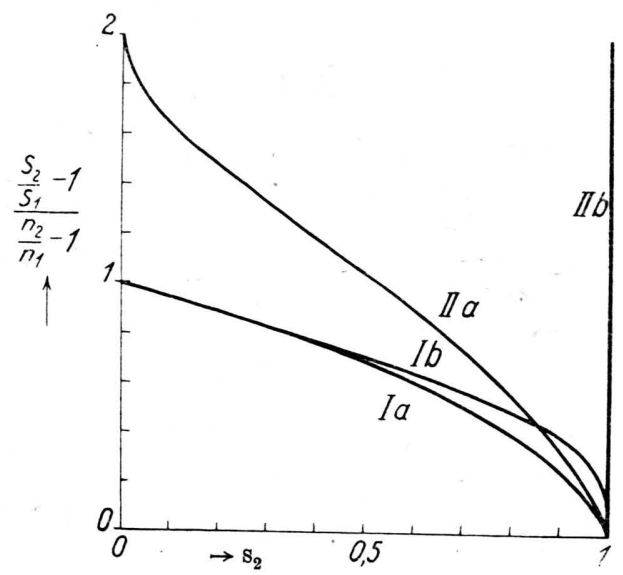

Abb. 7. Das Schwärzungsverhältnis $s_{2} / s_{1}$ bei konstantem Mischungsverhältnis $n_{2} / n_{1}$ als Funktion der Schwärzung $s_{2}$ bei Vorliegen der Schwärzungskurven $s=1-\exp (-n)$ (I) und $s=1-(1+n) \exp (-n)$

(II), wenn $n_{1} \approx n_{2}$ (a) und $n_{1} \ll n_{2}$ (b) ist.

man in diesem Gebiet mit einer geradlinigen Extrapolation auf die Schwärzung Null keinen großen Fehler begeht. (Man findet $n_{2} / n_{1}-1 \mathrm{um}$ höchstens $3 \%$ zu groß.)

Hrn. Prof. J. Mattauch, in dessen Abteilung ich die Untersuchung durchführen durfte, möchte ich für sein freundliches Interesse an der Arbeit und Frl. Scheld für ihre geschickten Dienste als Assistentin herzlich danken.

\title{
Ionisierungsspannung und Elektronenabschirmung im Periodischen System
}

\author{
Von Wolfgang Finkelnburg ${ }^{1}$
}

Herrn Prof. Dr. Hans Rau in Darmstadt zum 65. Geburtstag gewidmet

(Z. Naturforschg. 2 a, 16-20 [1947]; eingegangen am 9. Sept. 1946)

Die Abschirmung der auf das äußerste Elektron wirkenden Kernladung durch die inneren Elektronen zeigt im Periodischen System der Elemente Gesetzmäßigkeiten, die Schlüsse auf die Verhältnisse in der Elektronenhülle gestatten und die Extrapolation noch unbekannter sowie die Korrektur unsicherer Ionisierungsspannungen ermöglichen.

$\mathrm{D}^{\mathrm{i}}$ ie Ionisierungsenergie $E_{i}$ des im $n$-quantigen Zustand gebundenen Elektrons eines wasserstoffähnlichen Ions der Kernladung $Z e$ ist gleich dem Termwert des entsprechenden Energiezustands

\footnotetext{
1 z. Zt. Nördlingen/Bayern, Oskar-Mayer-Str. 10.
}

$$
E_{i}(n)=Z^{2} R / n^{2},
$$

wo $R$ die hier in $e$-Volt ausgedrückte $\mathrm{R} \mathrm{y} \mathrm{d} \mathrm{berg-}$ Konstante

$$
R=15,54 e-\text { Volt }
$$

ist. Bei einem beliebigen Atom der Ordnungszahl $N=Z$ wird ein Teil der Kernladung $Z$ in seiner 